\title{
Malignant tumors of the epipharynx - our five years of experience
}

\author{
Iliev G. ${ }^{1}$, Ivanovska Hr. ${ }^{2}$, Encheva E. ${ }^{2}$, Ivanova P. ${ }^{1}$, \\ Kerimov K. ${ }^{1}$, Milkov M. ${ }^{3}$ \\ 'ENT Department, St. Marina University Hospital, Medical University of Varna, Bulgaria \\ ${ }^{2}$ Radiotherapy Department, St. Marina University Hospital, Medical University of Varna, Bulgaria \\ ${ }^{3}$ Faculty of Dental Medicine, Medical University of Varna
}

\begin{abstract}
Introduction: The nasopharyngeal carcinomas are malignant neoplasms arising from the epithelial cells that cover the surface of the nasopharynx. Due to its asymptomatic presence this cancer is often diagnosed in the late stages of the disease. Options for treatment are radiotherapy and chemotherapy.

Materials and methods: A retrospective study over a period of 6 years presenting the cases of 45 patients with malignant tumors of the epipharynx and their follow-up.

Results: Thirteen of the all forty-five patients diagnosed with the nasopharyngeal carcinoma were admitted to the radiotherapy department. The results show a complete response in seven of the patients.

Conclusion: The malignant tumors of the epipharynx are a rare disease which still remain a late-on diagnosed tumor. Radiotherapy with concomitant chemotherapy has increased survival.
\end{abstract}

\section{Introduction}

The nasopharyngeal carcinomas are malignant neoplasms arising from the epithelial cells that cover the surface of the nasopharynx. Usually the nasopharyngeal cancer is asymptomatic in its initial stages and often presents with symptoms from adjacent organs, in the cervical area, frequently also with distant metastases. These tumors are more common among males. Etiological factors include Epstein-Barr virus (EBV), smoking, environmental factors, genetic predisposition. The nasopharyngeal tumor is not amenable to surgery, the treatment options are chemotherapy and radiotherapy.

Materials and methods Over a period of 6 years from 2012 to March 2018, 45 patients were diagnosed with nasopharyngeal carcinoma from a total of 711 proven head and neck cancers in the ENT Department UMHAT Saint Marina, Varna, Bulgaria. This defines a portion of $6,5 \%$ of all neoplasms diagnosed in the department. On the table below the results are shown by years: 


\begin{tabular}{|c|c|c|}
\hline Year & $\begin{array}{c}\text { Number of } \\
\text { patients with } \\
\text { nasopharyngeal } \\
\text { carcinoma }\end{array}$ & $\begin{array}{c}\text { Total number of } \\
\text { diagnosed cancers } \\
\text { in the head and neck } \\
\text { region }\end{array}$ \\
\hline 2012 & 10 & 98 \\
\hline 2013 & 5 & 120 \\
\hline 2014 & 9 & 103 \\
\hline 2015 & 6 & 112 \\
\hline 2016 & 6 & 153 \\
\hline 2017 & 7 & 98 \\
\hline 2018 & 2 & 27 \\
\hline
\end{tabular}

The gender incidence is in favor of males affecting 40 men or $89,9 \%$ and 5 women or $11,1 \%$ from the diagnosed patients in the clinics for the whole period. The youngest female patient was 41 years old and on the other hand the oldest -70 years old, concerning the male patients: youngest -24 years old and oldest -89 years old.

In three of our patients the tumor was diagnosed on the posterior wall of the nasopharynx, while in six patients the malignant process was affecting the lateral wall, in one patient the cancer was localized on the anterior wall, and in the other thirty-three patients the cancer was involving more than one wall of the epipharynx.

All patients were presented on onco-comitee after $\mathrm{PET} / \mathrm{CT}$ staging for determination the subsequent treatment.

Over the period $01.2015-03.2018$ in the radiotherapy department of UMHAT Saint Marina, Varna, thirteen of these patients diagnosed with nasopharyngeal carcinoma were admitted for treatment. Four of the patients were assigned to definitive radiotherapy (three patients in the second stage and one patient in the fourth stage) the remaining nine (six of them in the third stage and three in the fourth) were assigned to definitive radiotherapy with concomitant chemotherapy.

The early results were evaluated on a PET/CT basis six months after the end of treatment and the assessment of the local status with endoscopy.

\section{Discussion}

Almost all (approximately 98\%) of the tumors of the epipharynx are epithelial in origin - spinocellular, non-keratinizing, undifferentiated carcinoma (Lymfoepitelioma of Regaud - Schmincke 30$50 \%$ ). The second large group is lymphomas with a frequency of about $7 \%$ and most rarely are adenocarcinomas, sarcomas and melanomas.
Many theories are speculated about the etiology of these malignant neoplasms but it can be generally concluded that the etiology is still unclear. The nasopharyngeal carcinoma (NPC) is caused by a combination of factors - viral, environmental, chemical, mechanical, hereditary. From all the different causes of malignant nasopharyngeal tumors in the literature has been emphasized on the viral influence of the infection with Epstein-Barr virus (EBV).

The incidence of nasopharyngeal carcinomas in Europe and the USA is much lower than in South China, Southeast Asia and North Africa (the Maghreb region). Less than one out of every 100 , 000 people in North America suffers from nasopharyngeal carcinoma according to the American Cancer Society, while the incidence in Southeast Asia and North Africa is between 8-25/100,000. Survival is between $40-50 \%$ for isolated treatment solely with radiotherapy and $55-80 \%$ for combined chemotherapy and radiotherapy. According to Radiotherapy Society in Bulgaria epipharyngeal carcinoma's incidence in Bulgaria varies between about 0.5 per 100,000 people. Following the data in 2006 the mortality is respectively 24 men (52\%) and 3 women (14\%), as about $60 \%$ of the patients have been diagnosed in the III-rd and IV-th clinical stages.

According to the Radiotherapy Society in Bulgaria $80 \%$ of patients are already affected with regional metastases when diagnosed. Between $7 \%$ and $18 \%$ of the patients develop distant metastases most commonly in the lungs, liver and the bones.

\section{Results}

The results show a complete response in seven of the patients: three of them in the second stage of the disease, treated with radiotherapy only, and in the rest four patients successful treatment was achieved with combined radiotherapy and chemotherapy. One of the patients did not complete the full course of the designated treatment by his own will in 2015, in two patients improvement was observed; one patient died of comorbidity; and one did not appear to evaluate the treatment; the last patient is awaiting PET/CT staging.

\section{Conclusion}

From the reviewed literature and the followed-up patients, although in short-terms, the following conclusions can be drawn: 
- The malignant tumors of the epipharynx are a rare disease, affecting almost exclusively males with occurrence in all ages.

- The etiology of the neoplasm in still unknown, however, the cancer has been strongly linked to the Epstein-Barr virus infection.

- Nasopharyngeal carcinoma remains a late-on diagnosed tumor mostly in the third and fourth stage, often as a random finding in the diagnosis or treatment of other complaints or diseases.

- The results that were achieved with radiotherapy emphasize on the need for early diagnosis, and the combination with chemotherapy gives hope for complete remission in advanced tumors.

\section{References:}

1. Plamen Nedev, Otorhinolaryngology, 2008

2. Radiotherapy Association Society in Bulgaria, Nasopharyngeal Carcinoma, National standard for radiotherapy of the epipharyngeal cancer, 2009

3. Kutsarov A., Marinov Tsv., Rare clinical case of a young man with Shmincke tumor, Med info, issue 3, 2016

4. Petkov N., Melamed R., Nasopharyngeal Tumors, GP News, issue 3, 2005 